direct ventral tracts are much larger than the other two and can be traced as far as the lower sacral segments. The crossed lateral and direct dorsal seem to disappear in the upper thoracic region. Arranged according to the number of fibers which they contain, the order is crossed dorsal, direct ventral, crossed lateral, direct dorsal.

\title{
5 (7or)
}

Interpolated extra-systoles, of frequent occurrence, in an otherwise normal human heart.

\section{By M. DRESBaCH and S. A. MUNFord.}

[From the Physiological Laboratory, Cornell University, Ithaca, $N . Y$.]

In the extensive literature upon extra-systoles about twenty writers have described the type known as the interpolated beat of the ventricle. Although such beats have frequently been produced experimentally, their occurrence clinically is comparatively rare. ${ }^{1}$ Almost without exception they have been observed in cases showing gross lesions of the heart. The present case is of interest because the interpolated contractions occur persistently and frequently in a heart which, aside from a slow sinus rhythm, is otherwise normal. It furnishes a striking example of an organ on the borderland between a physiological and a strictly pathological condition.

T., a Chinese student at Cornell University, is in good health. $\mathrm{He}$ is able to take vigorous exercise, such as running, tennis, etc. About two years ago a cardiac irregularity was discovered in his routine physical examination. Prior to that time he was wholly ignorant of any disturbance in his heart, and is at no time conscious of the heart's contractions. Polygraphic records have frequently been made during the past two years and the organ has been found beating normally on two occasions only, and but for a short time.

The tracings show extra-systoles of the ventricle which occur with considerable regularity on some days, but usually they appear at varying intervals. The normal rate of the dominant rhythm

1 Mackenzie, Lewis, Wenckebach, and others. 
averages about 6o. It may go as low as 50 during rest. The extra-systoles average about 35 per minute during rest and 45 after vigorous exercise. The ectopic beat follows the normal contraction by a period averaging $1 / 2$ second. The pause following the extra-systole is variable; occasionally it is fully compensatory. The dominant rhythm is but slightly disturbed, as a rule. Often it is strikingly constant, and it is seldom more variable than the rhythm of many normal hearts. This is remarkable in view of the fact that in the tracings there is good evidence of retrograde contractions of the auricle which beats prematurely in response to the heterogenetic stimulus. Evidence for the retrogression is found in the absence of the " $a$ " wave at certain cycles. In place of the " $a$ " wave there is often seen a wave which precedes the normal auricular systole by $3 / 30$ to $5 / 30$ of a second. This premature wave is also preceded by a wave practically synchronous with the extra-systole of the ventricle.

The interpretation of the curves has been uncertain because of the unusual character of the phlebograms and cardiograms and because of the difficulty in establishing proper time relations. While several explanations may be offered for the type of irregularity exhibited, the evidence points to the one given as plausible, but not altogether satisfactory.

Physiologically this heart is interesting because of the following characters:

I. It very rarely has periods in which its action is absolutely normal. These periods are of short duration.

2. The normal sinus rate is slow.

3. Functionally the heart meets all ordinary demands made upon it.

4. There is a bathmotropic disturbance which causes persistent interpolated extra-systoles.

5. The number of extra-systoles is not decreased, except slightly in a few tracings, by an increase in the heart rate due to exercise. The tendency is for the number to rise with acceleration of the normal beat.

6. The pause following the extra-systole is variable. Occasionally it is compensatory.

7. There is strong evidence that the extra-systole of the 
ventricle is often followed by a premature beat of the auricle. When the evidence of such retrograde contractions is best, the indicated time of backward conduction is $7 / 30$ to $8 / 30$ of a second.

8. There is no evidence of lengthened $A s-V s$ intervals except at the extra-systoles.

9. There is a fairly fixed time relation between the normal and abnormal ventricular systoles.

10. There is often a considerable delay in the appearance of the carotid pulse resulting from the extra-systole.

II. There is a marked lack of synchronism between the carotid pulse and the " $c$ " wave. The latter may precede the former by $I / 20$ of a second.

12. The site of the abnormal stimulus is either in the ventricle or some part of the conduction system, e. g., the node, or some lower point.

$$
6(702)
$$

On the differences in the effects of stimulation of the two vagus nerves on rate and conduction of the dog's heart.

By ALFRED E. CoHN.

[From the Hospital of the Rockefeller Institute for Medical Research, New York.]

The effects obtained in the action of dogs' hearts on stimulating the peripheral stumps of both vagus nerves were studied in fiftyfour experiments. The dogs were anesthetized with ether without adjuvant, artificial respiration was maintained by the MeltzerAuer method, and registration was accomplished by the galvanometric method. When the chest was opened, curves of auricular and ventricular contractions were also inscribed. Faradic stimulation was employed. The secondary coil of an inductorium, fed by a 2-volt dry cell, placed arbitrarily at $50 \mathrm{~mm}$., was the source of the current.

On stimulating the right vagus nerve, the usual effect was obtained; both auricles and ventricles ceased to beat, generally throughout the period of stimulation. Occasionally the ventricles escaped from inhibition, but then the impulse to contraction arose 